\title{
Septic Cavernous Sinus Thrombosis: A Case Report
}

\author{
Mahdieh Arian, ${ }^{1}$ Azadeh Kamali, ${ }^{1,}{ }^{*}$ Mahbubeh Tabatabaeichehr, ${ }^{2}$ and Parisa Arashnia ${ }^{3}$ \\ ${ }^{1}$ Department of Nursing, Faculty of Nursing and Midwifery School, North Khorasan University of Medical Sciences, Bojnurd, IR Iran \\ ${ }^{2}$ Department of Midwifery, Faculty of Nursing and Midwifery School, North Khorasan University of Medical Sciences, Bojnurd, IR Iran \\ ${ }^{3}$ Imam Reza Hospital, North Khorasan University of Medical Sciences, Bojnurd, IR Iran \\ "Corresponding author: Azadeh Kamali, Department of Nursing, Faculty of Nursing and Midwifery School, North Khorasan University of Medical Sciences, Bojnurd, IR Iran. Tel: \\ +98-5832296909, E-mail: azadehkamali58@yahoo.com
}

Received 2015 December 15; Revised 2016 March 16; Accepted 2016 April 11.

\begin{abstract}
Introduction: Septic cavernous sinus thrombosis (CST) is a rare condition that can result in high mortality and morbidity rates if not treated immediately. CST may be aseptic or septic. Less common primary sites of infection include the tonsils, soft palate, middle ear, and orbit. Reported cases of middle ear infection are very rare, and response to treatment is poor.

Case Presentation: The present study is a case report of acute otitis media which led to septic cavernous sinus thrombosis in a 56-year-old woman in Bojnord city, North Khorasan, Iran.

Conclusions: Findings of laboratory tests and magnetic resonance imaging (MRI) confirmed the clinical diagnosis. Clinical-based medical care led to successful management of the patient with broad spectrum intravenous antibiotics that prevented serious complications.
\end{abstract}

Keywords: Septic Cavernous Sinus Thrombosis, Otitis Media, MRI Brain, Intravenous Antibiotics

\section{Introduction}

Cavernous sinus thrombosis (CST) was first described by Debase in 1778 (1). The primary source of CST may be a distant focus, with septicemia preceding thrombosis of the cavernous sinus. Alternatively, infection may spread from the facial regions, via the facial venous plexus or from the sphenoid sinus, directly to the adjacent cavernous sinus (2). Infection from these sites may easily travel to the cavernous sinus and cause thrombosis and occlusion, leading to serious implications involving the brain and eyes (3). Nasal furuncle is believed to be the most common cause of CST (50\%), followed by sphenoidal or ethmoidal sinuses (30\%) and dental infections $(10 \%)(4,5)$. Less common primary sites of infection include (in order of frequency) the tonsils, soft palate, middle ear, and orbit (orbital cellulitis). The highly anastomotic venous system of the paranasal sinuses allows retrograde spread of infection to the cavernous sinus via the superior and inferior ophthalmic veins $(6,7)$. Staphylococcus aureus is the most common infectious microbe, found in $70 \%$ of cases (8). Streptococcus is the second leading cause. Gram-negative rods and anaerobes may also lead to cavernous sinus thrombosis. Rarely, Aspergillus fumigatus and mucormycosis cause $\operatorname{CST}(5)$.

The case presented in this study is one of the few reported occurrences of such an event associated with septic CST that was caused by Staphylococcus aureus, secondary to acute otitis media. The objective of the present report is to familiarize clinicians with the clinical features, pathogenesis, diagnosis, and appropriate management of septic CST.

\section{Case Presentation}

A 56-year-old woman was admitted to the emergency ward with the chief complaint of fever with rigors, low level of consciousness, unilateral bulging right eye, inability to walk, and loss of vision, all of which had sudden onset on April 22, 2015. Her family gave a patient history that described her as a resident of a rural village near Bojnord city in North Khorasan, Iran. Two weeks prior to admission, on April 8, the patient experienced headache, severe earache, and throat pain, followed by fever and chills, which ultimately compelled her to visit a general physician in a rural health center. Temporomandibular joint pain drastically increased within a few hours, and an inflamed tympanic membrane confirmed an acute otitis media diagnosis by the doctor. Treatment was started with antibiotics (amoxicillin $500 \mathrm{mg}$ TDS) and analgesics/antipyretics (paracetamol). Clinical manifestations subsided for two days, but severe headache, anorexia, and high fever appeared again, followed by confusion.

These symptoms impelled the doctor to urgently refer the patient to the Imam Reza governmental specialized teaching hospital, which was the nearest internal disease 
center that also provides internal services including emergency, neurology, ICU, and specialist clinics. Physical examination of the woman at the time of arrival revealed a reduced level of consciousness. The patient was irritable, pale, and feverish (axillary temperature of $39.5^{\circ} \mathrm{C}$ ), with a pulse rate of $78 /$ minute, blood pressure $130 / 70 \mathrm{mmHg}$, respiratory rate of $21 /$ minute, and blood sugar measured by glucometer at $221 \mathrm{mg} / \mathrm{dl}$. Head and neck examination revealed severe unilateral exophthalmos, chemosis, and proptosis (Figure 1).

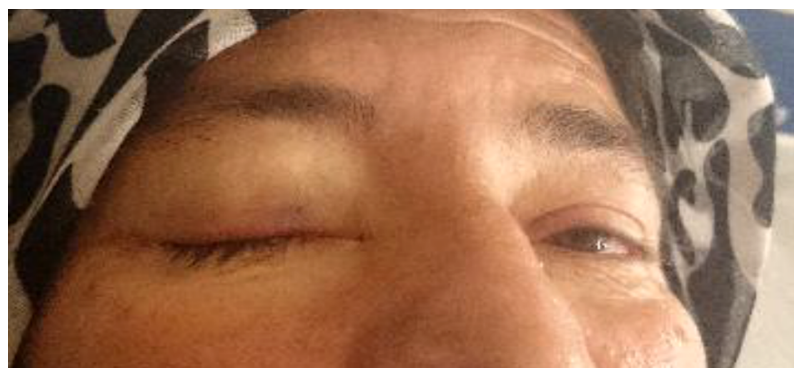

Figure 1. Swelling Over Right Eye Region, Erythema of the Lids, Inward Deviation of Eye with Diplopia and Gross Diminution of Vision

Intraoral examination was normal. Central nervous system examination revealed a yellow spot in the right eye and sluggish reaction to light in the right eye, while the left was reactive to light. Cranial nerve palsies of III and IV were present, whereas other cranial nerves were grossly intact, however with decreased power tone, reflexes, and positive meningeal signs (e.g., neck rigidity). Other neurological examinations could not be undertaken because the woman was semiconscious and irritable. Other examinations included lung: clear to auscultation bilaterally; abdomen: soft, non-tender, nondistended, normoactive bowel sounds; and heart: normal s1 and s2, no murmurs or gallops.

Other systems findings were non-contributory. The available laboratory tests revealed the following results. WBC: $8700 / \mathrm{mm}^{3}$, ESR: $50 \mathrm{~mm} / \mathrm{hr}, \mathrm{C}$-Reactive Protein positive $(+++)$, malaria test negative, brucellosis tests (Wright agglutination, Coombs Wright, and 2-Mercapto-Ethanol) negative, electrolytes: Na $131 \mathrm{mmol} / \mathrm{L}, \mathrm{K} 3.4 \mathrm{mmol} / \mathrm{L}$, urea 41 $\mathrm{mg} / \mathrm{dl}$, creatinine $1.3 \mathrm{mg} / \mathrm{dl}, \mathrm{SGOT}: 82 \mathrm{U} / \mathrm{L}, \mathrm{SGPT}: 46 \mathrm{U} / \mathrm{L}$, and FBS 96 mg/dl.

\subsection{Differential Diagnosis}

The provisional diagnosis was meningitis, orbital cellulitis, cavernous sinus thrombosis, and mucormycosis. The patient had no history of diabetes mellitus (DM), but her family reported her having had polyuria and polydipsia for two weeks prior to admission. While hospitalized, she was NPO over the last 24 hours, but her blood glucose level was $221 \mathrm{mg} / \mathrm{dl}$. On the second day after admission, she rapidly developed left proptosis with moderate chemosis. In addition, movement in both eyeballs was limited in all directions to about half the normal range, but vision and facial sensation were intact. Proptosis and periorbital swelling were present bilaterally, but more severe on the right side. On the third day, extraoral examination revealed a solitary swelling around the mouth and orbital cellulitis with pus discharge (Figure 2).

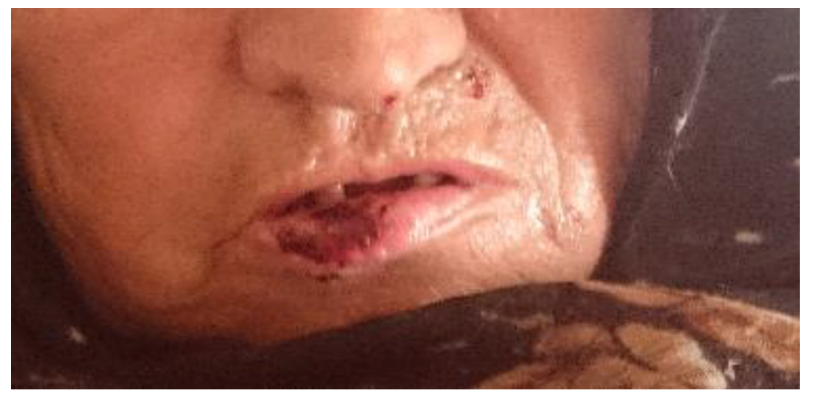

Figure 2. Extraoral Examination Revealed a Solitary Swelling Around the Mouth and Orbital Cellulitis with Pus Discharge

For immediate management, the patient received intravenous fluids (500cc glucose/saline), antibiotics (vancomycin 2gr BID, meropenem 2gr TDS, metronidazole 500mg QID, serum dextrose saline 1000cc + 5cc Kcl 15\%, amphotericin 50mg daily) intravenously. On intraoral examination, the maxillary palatal was grossly intact, and there was no gingival or mucosal denudation exposing the necrotic bone with pus exudation. Tenderness was not elicited on palpation. Hard tissue examination revealed no tenderness on percussion. Based on these findings, the provisional diagnosis of mucormycosis was rejected. For immediate management, the patient received mupirocin topical 2\% TDS, vitamins A and D TDS, and Nilstat Oral Drops (Nystatin) 20 drops TDS.

After mucormycosis was rejected, the patient was referred for a computerized tomography (CT) scan, an MRI, magnetic resonance imaging venography (MRV), and blood tests. Blood culture was positive for Staphylococcus aureus. MRI and MRV performed with the clinical suspicion of cavernous sinus thrombophlebitis (CST) demonstrated the following results: engorgement of the right superior ophthalmic vein and hyperintense signal changes of the right temporal and inferior frontal areas. On long TR, sequences were seen, as well as signal changes in the right basal ganglia. Spread Cavernous sinus thrombosis was caused, Restricted ischemic focal on the frontal, temporal and basal ganglia areas. Brain MRI confirmed the cav- 


\begin{tabular}{|c|c|c|}
\hline Physical examination & \multicolumn{2}{|c|}{ Findings } \\
\hline Central nervous system examination & \multicolumn{2}{|c|}{$\begin{array}{l}\text { 1. Yellow spot in the right eye, } 2 \text {. Sluggish reaction to light in the right eye, } 3 \text {. III and IV cranial nerve palsies, } 4 \text {. Decreased } \\
\text { power tone, } 5 \text {. Decreased reflexes, } 6 \text {. Positive meningeal signs (neck rigidity) }\end{array}$} \\
\hline Lung & \multicolumn{2}{|c|}{ Clear to auscultation bilaterally } \\
\hline Abdomen & \multicolumn{2}{|c|}{ Soft, non-tender, nondistended, normoactive bowel sounds } \\
\hline Heart & \multicolumn{2}{|c|}{ Normal s1 and s2, no murmurs or gallops } \\
\hline \multicolumn{3}{|l|}{ Table 1. Laboratory Test Results } \\
\hline Laboratory tests & & Results \\
\hline WBC & & $8700, \mathrm{~mm}^{3}$ \\
\hline ESR & & $50, \mathrm{~mm} / \mathrm{hr}$ \\
\hline C-Reactive Protein & & Positive, $(+++)$ \\
\hline Malaria test & & Negative \\
\hline \multicolumn{2}{|c|}{ Brucellosis tests (Wright Agglutination, Coombs Wright and 2-Mercapto-Ethanol) } & Negative \\
\hline $\mathbf{N a}$ & & $131, \mathrm{mmol} / \mathrm{L}$ \\
\hline $\mathbf{K}$ & & $3.4, \mathrm{mmol} / \mathrm{L}$ \\
\hline Urea & & $41, \mathrm{mg} / \mathrm{dl}$ \\
\hline Creatinine & & $1.3, \mathrm{mg} / \mathrm{dl}$ \\
\hline SGOT & & $82, \mathrm{U} / \mathrm{L}$ \\
\hline SGPT & & $46, \mathrm{U} / \mathrm{L}$ \\
\hline FBS & & $96, \mathrm{mg} / \mathrm{dl}$ \\
\hline
\end{tabular}

ernous sinus thrombosis. The patient responded well to broad spectrum intravenous antibiotics (meropenem $2 \mathrm{gr}$ TDS, vancomycin 1gr BD, and metronidazole 200mg) and supportive treatment. Follow-up MRI was performed. After intravenous antibiotic therapy, all abnormalities resolved, aside from the III and IV cranial nerve pareses, which were unchanged at discharge one month later.

\section{Discussion}

The cavernous sinus, which is anatomically placed in the posterior portion of the optic canal and superior to the orbital fissure, is a vital structure as a pathway for the internal carotid artery and cranial nerves III, IV, V, and VI. Thus, any infection or thrombosis in this area could be followed by a group of clinical manifestations including ptosis, ophthalmoplegia, diplopia, and paresthesia around the orbital cavity due to involvement of the eye motor nerves and impairment in the ophthalmic and maxillary branches of cranial nerve V (1). Tributaries of superior and inferior ophthalmic veins directly drain to the cavernous sinus, thus proptosis and chemosis themselves may lead to drain blockage of the ophthalmic veins (9). Complex anastomosis of the cavernous sinus between the facial veins with no valve and the pterygoid plexus, which is located very close to it, and also contralateral and intercavernous sinus connections could be a logical reason for the appearance of orbital symptoms on both sides, or on the opposite side $(1,10$, 11).

A decreased rate of cavernous sinus thrombosis mortality has been reported from $100 \%$ in the era preceding availability of antibiotics to 20\% since 1940 (12). In addition, mortality reduction from $50 \%-75 \%$ to $22 \%$ has been pointed by Yarington (13). This reduction occurred despite the threat of temporary complications and long-term sequelae (13).

The diagnosis of CST is based on clinical findings and confirmed by appropriate radiographic studies. Contrastenhanced CT scan can be helpful in determining the primary source of infection, superior ophthalmic vein thickening, and irregular filling defects in the cavernous sinus. Magnetic resonance imaging and venogram are more sensitive methods which have been proposed to reveal flow parameters. Findings may include a heterogeneous signal 
from the abnormal cavernous sinus and hyperintense signal of the thrombosed vascular sinuses, along with deformity of the cavernous portion of the internal carotid artery.

Clinical manifestations play a considerable role in diagnosis of CST. Prompt treatment of septic CST, while required, depends on the patient's condition and is best administered with high-dose, broad spectrum, intravenous antibiotic therapy in a period of three to four weeks (8).

\subsection{Conclusion}

In $70 \%$ of sinus thrombosis cases, the sagittal sinus, which is responsible for venous drainage of a large portion of the cerebral cortex and absorption of cerebrospinal fluid, is involved. Single involvement of the lateral sinus thrombosis is rare, although it mostly occurs along with involvement of other sinuses, mainly due to otitis media. While rarely leading to death, cavernous sinus thrombosis has the highest rate of mortality. Rarely, middle ear infection can be a cause of septic cavernous sinus thrombosis, and response to treatment is poor. Early diagnosis can be achieved with precise diagnostic methods such as MRI and MRV. Moreover, severe, irreversible, and fatal side effects of the disease can be prevented by early treatment, which can lead to successful management of patients $(14,15)$.

\section{Footnote}

Authors' Contribution: Acquisition, analysis, and interpretation of data and drafting of the manuscript, Mahdieh Arian, Azadeh Kamali, Mahbubeh Tabatabaeichehr, Parisa Arashnia.

\section{References}

1. Cho JY, Kim HM, Ryu JY. Cavernous sinus thrombosis progression from trismus.J Korean Assoc Oral Maxillofac Surg. 2015;41(1):43-7. doi: 10.5125/jkaoms.2015.41.1.43. [PubMed: 25741468].
2. Lai PF, Cusimano MD. The spectrum of cavernous sinus and orbital venous thrombosis: a case and a review. Skull Base Surg. 1996;6(1):539. [PubMed: 17170953].

3. Raghunandan K, Shetkar UB, Somen M, Rajen M. Septic cavernous sinus thrombosis-A case report. Pravara Med Rev. 2011;6(2):37-9.

4. Pavlovich P, Looi A, Rootman J. Septic thrombosis of the cavernous sinus: two different mechanisms. Orbit. 2006;25(1):39-43. doi: 10.1080/01676830500506077. [PubMed:16527775].

5. Ferri FF. Ferri's Clinical Advisor 2016: 5 Books in 1. Philadelphia: Elsevier Health Sciences; 2015. p. 1133.

6. Zhang J, Stringer MD. Ophthalmic and facial veins are not valveless. Clin Experiment Ophthalmol. 2010;38(5):502-10. doi: 10.1111/j.14429071.2010.02325.x. [PubMed: 20491800].

7. Sharouny H, Narayanan P. Endoscopic marsupialisation of the lateral frontal sinus mucocele with orbital extension: a case report. Iran Red Crescent Med J. 2015;17(1):17104. doi: 10.5812/ircmj.17104. [PubMed: 25763256].

8. Kiddee W, Preechawai P, Hirunpat S. Bilateral septic cavernous sinus thrombosis following the masticator and parapharyngeal space infection from the odontogenic origin: a case report. J Med Assoc Thai. 2010;93(9):1107-11. [PubMed: 20873087].

9. Horowitz A, Spendel D, Kraut R, Orentlicher G. Cavernous sinus thrombosis as a result of a fungal infection: a case report. J Oral Maxillofac Surg. 2013;71(11):1899. doi: 10.1016/j.joms.2013.05.021. [PubMed: 23945514].

10. Watkins LM, Pasternack MS, Banks M, Kousoubris P, Rubin PA. Bilateral cavernous sinus thromboses and intraorbital abscesses secondary to Streptococcus milleri. Ophthalmology. 2003;110(3):569-74. doi: 10.1016/S0161-6420(02)01765-7. [PubMed: 12623823].

11. Goawalla A, Mansell N, Pearson A. Septic cavernous sinus thrombosis with bilateral secondary orbital infection. Orbit. 2007;26(2):113-6. doi: 10.1080/01676830600675418. [PubMed: 17613858].

12. Southwick FS, Richardson EP, Swartz MN. Septic thrombosis of the dural venous sinuses. Medicine (Baltimore). 1986;65(2):82-106. [PubMed: 3512953].

13. Yarington CT. Cavernous sinus thrombosis revisited. Proc R Soc Med. 1977;70(7):456-9. [PubMed: 331338].

14. Bousser MG, Barnett HJM. Cerebral venous thrombosis. In: Barnett HJM, Mohr JP, Stein BM, editors. Stroke. 3. Philadelphia: Churghill- Livingstone; 1998 . pp. 623-42.

15. Fishman RA. Cerebral veins and sinuses. In: Rowland LP, editor. Merriit Neurology. 11. Philadelphia: Lippincott WillIams \& Wilkins; 2005. pp. 338-41. 